Address for Correspondence:

Dr. Andrea Mancuso, MD, Medicina

Interna 1, ARNAS Civico, Piazzale Leotta

4, 90100, Palermo, Italy.

Email: mancandrea@libero.it

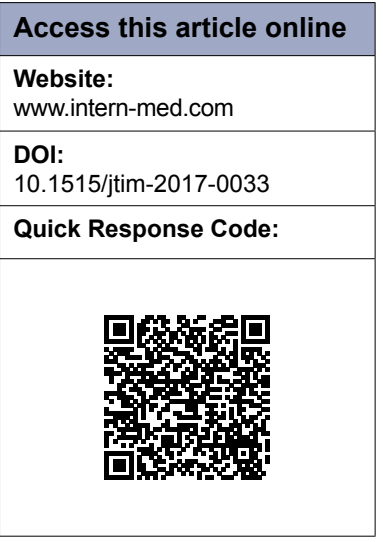

\title{
Timing of transjugular intrahepatic portosystemic shunt for Budd-Chiari syndrome: An Italian hepatologist's perspective
}

\author{
Andrea Mancuso ${ }^{1,2}$ \\ 'Medicina Interna 1, ARNAS Civico - Di Cristina - Benfratelli, Piazzale Leotta 4, Palermo, Italy; \\ Epatologia e Gastroenterologia, Ospedale Niguarda Ca' Granda, Piazza Ospedale Maggiore 3, 20162 Milano, Italy
}

\section{ABSTRACT}

Budd-Chiari syndrome (BCS) management flow-chart is derived from experts' opinion and is not evidence-based. Guidelines suggest BCS management should follow a stepwise strategy: medical therapy as first-line treatment, revascularization or transjugular intrahepatic portosystemic shunt (TIPS) if no response to medical therapy, and liver transplant as rescue therapy. Recent evidence suggests that only medical therapy results in a bad long-term outcome. The biggest criticism of guidelines is the indication that BCS should receive further treatment only when hemodynamic consequences of portal hypertension become clinically evident. Recent data support that in BCS liver fibrosis could arise from chronic microvascular ischemia. A reasoning model of BCS physiopathology is that impaired hepatic vein outflow has hemodynamic consequences on portal hypertension development and causes hepatic fibrosis and liver failure through chronic ischemic damage. On this assumption is the concept that relieving liver congestion could ameliorate liver function and prevent development of BCS complications. Recently, early interventional treatment with TIPS for BCS has been reported to be effective. Early TIPS seems to be the best option for BCS management. Future multicenter controlled studies should compare the outcome of BCS treated with early interventional treatment compared with stepwise strategy.

Key words: Budd-Chiari Syndrome, transjugular intrahepatic portosystemic shunt (TIPS), liver transplant, outcome

\section{INTRODUCTION}

Budd-Chiari Syndrome (BCS) is a rare and severe thrombotic disease affecting hepatic veins (HVs) and/or inferior vena cava (IVC) and impairing hepatic outflow. Although many of the cases are idiopathic, one or more prothrombotic conditions are usually discovered, the most frequent of which are myeloproliferative diseases ${ }^{[1-4]}$. However, as it has been recently emphasized, the role of prothrombotic conditions seem to be relevant in the West, where BCS mostly involve HVs, while less in Asia, particularly in China, where the involvement of IVC is significant and the role of prothrombotic conditions, in particular of myeloproliferative diseases, is less frequent and other factors (congenital or infective?) seem to play an important role. As a consequence, albeit the considerations about physiopathology are similar, those about management could differ ${ }^{[5]}$.

The present editorial will concentrate on the management of BCS, in particular regarding complications of portal hypertension, prevention and treatment of liver failure (LF), which altogether represent the commonest topic of BCS management. However, other factors can affect BCS outcome. In fact, hepatocellular carcinoma (HCC) development is surely a concern during follow up of $\mathrm{BCS}^{[6]}$. Moreover, the outcome of underlying disease is other issue, since about $10 \%$ of the patients are reported to die because of hematological disease progression $^{[7,8]}$. Finally, both different sources of bleeding ${ }^{[9]}$ and thrombosis 
of other organ ${ }^{[8]}$ are further complications potentially affecting BCS outcome.

Since BCS is rare, both classification and management are empirical and not evidence-based, and follow opinion leaders' suggestion ${ }^{[1-4]}$. Simply, from a clinical point of view, apart from acute or fulminant cases, we should distinguish the two clinical phases ${ }^{[6]}$. The former is the asymptomatic phase (AP), in which either hepatic vein and/or IVC thrombosis is present but clinically silent: this likely represents the early phase of most of the patients with BCS. The latter is the symptomatic phase (SP), which could be divided into two forms: a chronic SP, in which signs of portal hypertension, mainly ascites, are evident while hepatic function is relatively preserved; an acute SP, in which liver failure develops. In fact, in most of the cases, clinical complications of BCS likely become evident after months or years after $\mathrm{HV}$ thrombosis has arisen, as either both abdominal and/or subcutaneous porto-systemic spontaneous shunts are often evident at diagnosis ${ }^{[3]}$. Progression from a phase to another could be correlated to temporally subsequent thrombotic events. However, since there is no strong evidence of a direct correlation between extension of thrombosis and BCS severity, a major role is probably played by hepatic functional reserve ${ }^{[7]}$.

Canonical pathway of hepatic fibrogenesis and cirrhosis postulate inflammation to be the driver of hepatic fibrogenesis in hepatitis. Due to the absence of inflammation, an ancillary hypothesis of parenchymal extinction was proposed in hepatic congestion ${ }^{[10,11]}$. An alternative theory, valid for both hepatitis and hepatic congestion, postulates that liver cirrhosis development could arise from chronic micro-vascular ischemia ${ }^{[12]}$. One model of this theory is BCS. In fact, a substantial rate of patients with BCS (numerically different in the cohorts of patients with BCS published) finally develops liver cirrhosis, in the absence of known causative factors but chronic liver ischemia ${ }^{[13]}$. This theory was not evidence-based, required validation and only pretended to serve as a basis for future research ${ }^{[14]}$. However, some confirmation was reported. In fact, in a murine experimental model of congestive hepatopathy through partial ligation of the inferior vena cava, researchers from Mayo Clinic, Rochester, showed that chronic hepatic congestion leads to sinusoidal thrombosis and strain, which in turn promote hepatic fibrosis ${ }^{[15]}$. Consequently, a reasoning model of BCS physiopathology is that not only impaired hepatic vein outflow has hemodynamic consequences on the development of portal hypertension, but also causes hepatic fibrosis development and liver failure through chronic ischemic liver damage ${ }^{[6]}$. This assumption is based on the concept that relieving liver congestion through treatment could ameliorate liver function and prevent development of BCS complications ${ }^{[15,16]}$.

\section{TREATMENTS FOR BUDD-CHIARI SYNDROME}

Both AASLD and EASL guidelines, expression of experts' opinion and not evidence-based experiences, suggest that BCS management should follow a stepwise management. In fact, medical therapy (anticoagulation, treatment of underlying disease, symptomatic therapy of portal hypertension complications) is suggested as first-line treatment, angioplasty or stenting as second-line (in patients with short-length stenoses not responding to medical therapy), TIPS the next step (in those not responding to medical therapy and in case of no response to, or stenosis unsuitable for, angioplasty or stenting) and liver transplant (LT) the last resor $\mathrm{t}^{[1]}$. However, although stepwise management suggests moving forward when no response to therapy appears, definition for response to therapy was not stated ${ }^{[1-4,6,15-17]}$ and a proposal of such a definition needs validation ${ }^{[18]}$.

Apart from medical therapy, that is, anticoagulation, which should probably be indicated as sole treatment only in cases without any sign of portal hypertension, BCS management relies on interventional or surgical approaches ${ }^{[1-4]}$.

In some experiences, short-length stenoses have been treated with angioplasty or stenting, with a good medium term outcome ${ }^{[19-20]}$.

The most frequent treatment for BCS is surely TIPS ${ }^{[1-4]}$. TIPS has been shown to be effective as the treatment for BCS in early experiences ${ }^{[21-23]}$ and can be successful also in the technically difficult case of extension of thrombosis to the portal vein tree ${ }^{[24,25]}$. Moreover, a multi-center experience provided long-term data on TIPS treatment for 147 BCS patients not responding to medical treatment or recanalization. TIPS was successful in 124 BCS patients, who were followed for a median of 36.7 months. Main complications were hepatic encephalopathy in $21 \%$ and TIPS dysfunction in $41 \%$ of the patients. The 10 year survival was $69 \%{ }^{[26]}$. Recently, wide single-centre experiences have confirmed an excellent outcome after $\operatorname{TIPS}^{[27-28]}$.

Apart from LT, surgical treatment is generally not contemplated in the management of BCS in recent guidelines ${ }^{[1-3]}$. In fact, the most used treatment for BCS nonresponsive to medical therapy is TIPS. LT is used as a rescue therapy, while surgical treatments are rarely contemplated ${ }^{[1-4]}$. Surgical shunts for BCS can be very successful, although they are associated with rapid decompensation and high in-hospital mortality (about 25\%), primarily due to the patients' poor general condition $^{[29-32]}$. Previously, surgery was considered the first 
choice. In fact, some series reported an excellent outcome after long-term follow-up ( $95 \%$ survival, 3 - to 28 -year follow-up ${ }^{[32]}$. However, in this series, the SSPCS was used, an approach that cannot be used in case of inferior vena cava (IVC) thrombosis or significant compression. In the same series, the mortality rates of patients with IVC involvement were very high after traditional surgery (mesoatrial shunts) and better results were reported using another technique (SSPCS + cavoatrial shunt) in 18 patients, all surviving after a follow-up of 5 to 25 years $^{[32]}$. However, outcome after surgical shunt is variable and worse results were reported by others ${ }^{[30,31,33]}$. In most of the above series, patients with liver failure were not considered for surgery but for liver transplantation ${ }^{[31-33]}$.

BCS due to a IVC obstruction near the right atrium constitutes a difficult to treat subgroup. In fact, management of BCS can benefit from endovascular dilatation or stenting, or surgical treatment. Obviously, TIPS does not bypass IVC obstruction, by giving an ineffective result. Endovascular management for patients with IVC obstruction near the atrium in reported to be safe and effective, but data are scanty and need confirmation on a larger scale ${ }^{[34]}$.

The widest experience with a surgical shunt approach for BCS due to a IVC obstruction was the above reported using a combination of SSPCS and cavo-atrial shunt: 18 patients, all surviving after a follow-up of 5 to 25 years ${ }^{[32]}$. Other surgical techniques reported in that and other series had roughly unacceptable outcomes when reported ${ }^{[30,31,33]}$. We recently described a patient with IVC obstruction near the atrium and a previous severe complication of a trial of endovascular management, who had an excellent outcome after the replacement of the obstructed segment of the IVC with a caval homograft ${ }^{[35]}$.

LT is the last chance for BCS syndrome management, in cases nonresponsive to either medical therapy or recanalization or decompression ${ }^{[36-43]}$. A European multi-center study reported long-term data on 248 patients who underwent LT for BCS. Hepatocellular carcinoma was incidentally found in explanted liver in three patients. Median follow-up was 48 months. Overall, $67(27 \%)$ patients died (49\% in the first month) because of sepsis in $47 \%$, graft dysfunction or hepatic artery thrombosis in $19 \%$, venous thrombosis in $12 \%$, cardiac complications in $9 \%$ and brain damage in $5 \%$. There was a significantly increased mortality if LT was shortly after SPSS or TIPS. Thirty-seven patients underwent re-LT (four of them twice). The 10-year survival was $68 \%$. After 1 year there were nine deaths, seven of which were in MPD patients. Complications post-OLT in the patients treated with anticoagulation were thrombosis in 27 patients (11\%), 11 of whom (41\%) died; recurrence of BCS in six (1 Re-OLT, 1 TIPS, 4 death); bleeding in $27(11 \%)$, two of whom died (intracranial bleeding). Prognostic factors were pre-OLT renal function and preOLT SPSS/TIPS ${ }^{[40]}$. However, the prognostic factor of a previous shunt before LT has to be weighed cautiously because it can only reflect the fact that patients who underwent TIPS before LT had the most severe liver disease. Moreover, a recent American multi-center study found no negative effect of TIPS on the following LT outcome $^{[41]}$. Finally, recent data show promising results after living donor LT for $\mathrm{BCS}^{[44]}$.

\section{CHOICE OF TREATMENT}

The treatment of choice for BCS with signs of portal hypertension should be TIPS ${ }^{[7]}$. The reasons are many. The former is that the technique is homogeneous and the procedure is the mostly used compared to both angioplasty or stenting and surgery. However, whether TIPS should be preferred to angioplasty or stenting in patients with short-length stenosis remains an unanswered question. Although no data can argue against the use of angioplasty or stenting in the subgroup with short-length stenosis, TIPS is probably a better choice because it gives assurance of a complete hepatic vein outflow relief, avoiding the concern of pre-procedure radiological under-estimation of the thrombosis extension. However, such a therapeutic choice in this subgroup of patients should be personalized and based on local expertise, since a prospective comparison between TIPS and angioplasty or stenting has not been performed. TIPS should be also preferred to surgery. In fact, surgical shunts techniques are many and with a wide heterogeneity in the outcome reported. Angioplasty or stenting should be the treatment of second line in the subgroup with short-length stenosis if TIPS is ineffective or in cases unsuitable for TIPS.

Surgical shunts should be treatment of choice when both TIPS and angioplasty or stenting are ineffective or unsuitable. LT has to be considered as a salvage treatment ${ }^{[7]}$.

\section{EARLY VERSUS DELAYED INTERVENTIONAL TREATMENT OF BCS}

AASLD guidelines for the stepwise management of BCS suggest moving forward when no response to therapy appears ${ }^{[1]}$, as I reported above. However, definition for response to therapy was not stated $\mathrm{d}^{[1-4,7,14-16]}$ and a proposal of such a definition needs validation ${ }^{[18]}$. Moreover, such proposal suggests arbitrary clinical criteria that are at the same way debatable and not easily usable in clinical practice (Figure 1). However, outcome is based on these criteria. 


\begin{tabular}{ll}
\hline Table 1: Clichy Definition for response to Budd-Chiari Syndrome treatment \\
Complete response & - No ascites \\
& - Normal Na and creatinine with no or low-dose diuretics (spironolattone $75 \mathrm{mg}$ or furosemide $40 \mathrm{mg} / \mathrm{die}$ ) \\
& - Factor $\mathrm{V}$ increase $>40 \%$ of the normal range \\
& - Bilirubin decrease $<15 \mathrm{mmol} / \mathrm{L}$ \\
& - No portal hypertension bleeding \\
& - No spontaneous bacterial peritonitis \\
& - BMl $>20 \mathrm{~kg} / \mathrm{m}^{2}$ \\
\hline Ongoing response & - Ascites detectable but responsive to low-dose diuretics \\
& - Normal Na and creatinine \\
& - Factor $V$ increase (if initially low) \\
Treatment failure & -Bilirubin decrease \\
\hline
\end{tabular}

BMI: body mass index

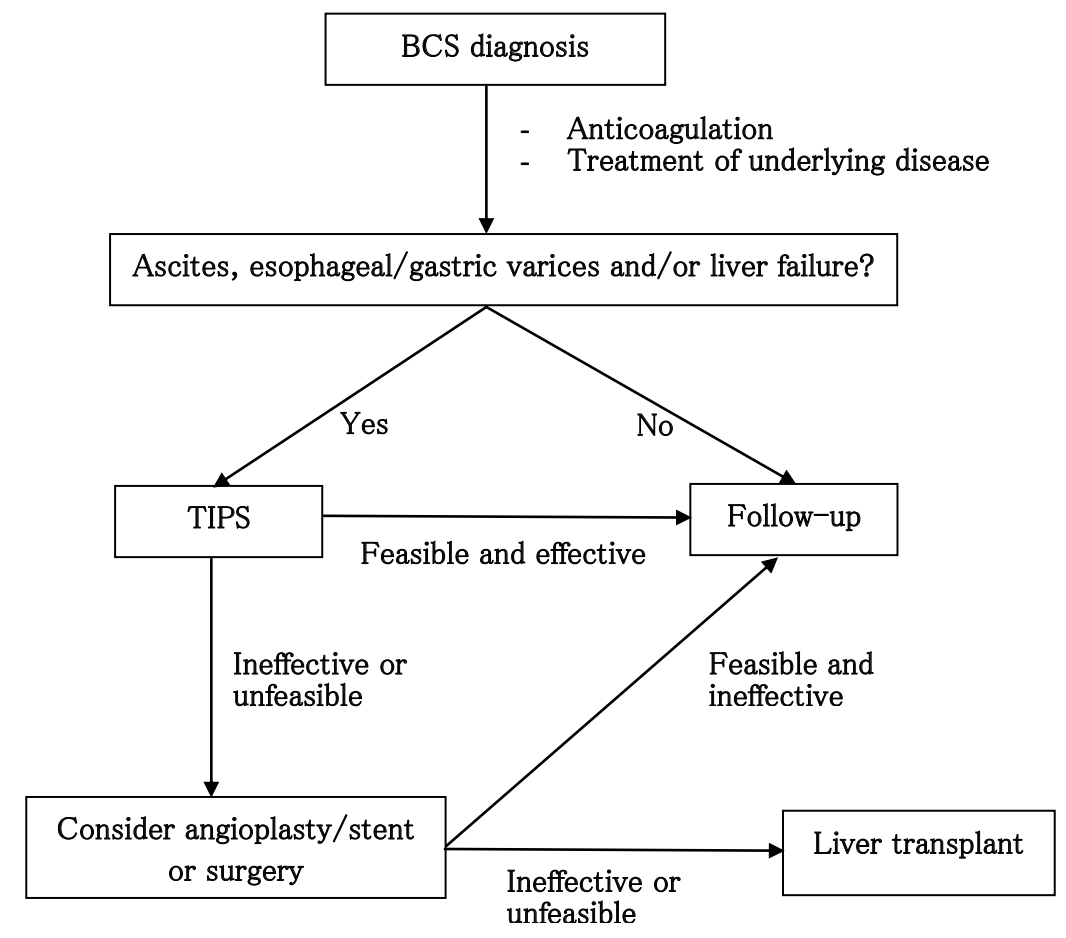

Figure 1: Proposal of early intervention for Budd-Chiari syndrome (BCS).

The outcome of BCS with currently available treatment is described in a prospective multi-center study in which 163 patients were followed for a median of 17 months (range 1-31 months); 18\% also had portal vein thrombosis, $84 \%$ had a thrombophilic syndrome, $46 \%$ of whom had a myeloproliferative disorder. Overall, 29 died: eight due to liver failure, two due to multiple organ failure, and two due to bleeding. The 24 -month survival was $82 \%$. Importantly, about one-third of the patients remained on medical therapy only, reporting a worse outcome ${ }^{[45]}$. However, the follow-up was not long enough to eventually show the consequences of a slowly progressing disease, possibly prevented by early recanalization or decompression, and to draw any definitive conclusion about the exact timing of treatment. The long-term outcome of the same multicenter experience was recently reported. Interestingly, 20 of the 69 patients on only medical therapy died, a high rate considering the availability of further invasive treatments. The remaining 88 underwent invasive treatment, mostly TIPS, 20 LT and only three surgical shunts. The overall survival was $74 \%$ at 5 years. However, a substantial rate of patients $(10 \%)$ died because of hematologic progression, suggesting that the outcome of the underlying disease can affect BCS outcome despite any BCS treatment ${ }^{[6]}$.

The biggest criticism of AASLD indication for the management of BCS is that they are based on the assumption that further treatment should be done for BCS only when hemodynamic consequences on portal hypertension become clinically evident, such as giving 
no consideration to the chronic ischemic liver damage effects on hepatic function and to the possibility of preventing liver failure by relieving impaired hepatic veins outflow $^{[3,5,12-14]}$. Moreover, patients receiving only medical therapy often have a bad outcome. In fact, the widest longterm multicenter experience on BCS reported that almost $30 \%$ of patients who received only medical therapy died, a high rate considering the availability of further effective invasive treatments ${ }^{[8]}$. Furthermore, a recent systematic review confirmed the bad outcome of BCS on only medical therapy, with survival worsening during long-term follow up in patients on only medical therapy ${ }^{[4]}$.

Recently, I reported a proposal of a new algorithm for the management of BCS (Figure 2), in which medical therapy is suggested only for patients without any sign of portal hypertension, whether early interventional treatment when either any symptom or sign of portal hypertension appears, with the aim of preventing hepatic fibrosis development, disease progression, and finally improving outcome $\mathrm{e}^{[3-7]}$. As I suggested, the same considerations about both timing and choice of treatment in BCS should probably apply to both adults and children ${ }^{[47,48]}$.

Recently, some confirmation about the opportunity of early TIPS for BCS has been reported ${ }^{[49,50]}$. In fact, a group from China reported data on 100 patients with BCS with diffuse occlusion of HVs out of 377 BCS from January 2007 to December 2010. Overall, 91 received early TIPS and nine refused TIPS (control group). Of the 91 patients who received TIPS, TIPS dysfunction was reported in 10 and treated effectively with balloon dilatation. Before TIPS hepatic encephalopathy (HE) was present in three patients, after HE in five (the $3+$ other 2 ), always mild. Survival data were the following: six out of 91 died in the early TIPS Group, eight out of nine died in the Control group. The authors concluded that TIPS is an effective and safe treatment for BCS with diffuse occlusion of HVs. Moreover, severe jaundice and pre-TIPS HE are not contraindications for TIPS in BCS and patients diagnosed with these features should undergo TIPS as soon as possible ${ }^{[49]}$. Furthermore, another group from Sweden reported data on 13 BCS patients treated with early TIPS. There were four cases of TIPS dysfunction, and three mild HE post-TIPS. Both one- and five-year transplantfree survival was $100 \%$ and $93 \%$, respectively. The authors concluded that TIPS is a safe and effective treatment for symptomatic BCS and should be considered the firstline intervention in patients with insufficient response to medical treatment ${ }^{[50]}$.

However, the assumption that relieving hepatic veins flow through TIPS or other interventional treatment could improve BCS outcome by solving ischemic injury remains to be proved. Nevertheless, it is highly probable that relieving outflow block has a positive role on necrosis and fibrosis in BCS. Moreover, although it is not clear whether cover TIPS relieves outflow block and indeed congestion is often present long after TIPS in BCS, it is possible that by simply inverting part of the flow through TIPS, such a partial reduction of congestion and hepatocellular edema could allow hepatic function improvement in cases with adequate hepatic functional reserve.

\section{CONCLUSION}

BCS is a challenging syndrome whose optimal management is still an open issue. Early TIPS seems to be the best option for BCS management. However, future multicenter controlled studies should compare the outcome of BCS treated with early interventional treatment compared with step-wise strategy.

\section{Disclosure}

I declare I have nothing to disclose.

\section{REFERENCES}

1. DeLeve LD, Valla DC, Garcia-Tsao G. Vascular disorders of the liver. Hepatology 2009; 49: 1729-64.

2. Mancuso A. Budd-Chiari syndrome management: lights and shadows. World J Hepatol 2011; 3: 262-4.

3. Mancuso A. An Update on Management of Budd-Chiari syndrome. Ann of Hepatol 2014; 3: 323-6.

4. European Association for the Study of the Liver; Garcia-Pagán JC; Buscarini E, Janssen HLA, Leebeck FW, Plessier A, Rubbia-Brandt L, et al. EASL Clinical Practice Guidelines: Vascular diseases of the liver. J Hepatol 2016; 64: 179-202.

5. Qi X, Han G, Guo X, De Stefano V, Xu K, Lu Z, Xu H, et al. The aetiology of primary Budd-Chiari syndrome: differences between the West and China. Aliment Pharmacol Therapeut 2016; 44: 1152-67.

6. Mancuso A. Management of hepatocellular carcinoma: Enlightening the gray zones. World J Hepatol 2013; 5: 302-10.

7. Mancuso A. An Update on Management of Budd-Chiari Syndrome: the Issues of Timing and Choice of Treatment. Eur J Gastroenterol Hepatol 2015; 27: 200-3.

8. Seijo S, Plessier A, Hoekstra J, Dell'era A, Mandair D, Rifai K, et al. Good long-term outcome of Budd-Chiari syndrome with a step-wise management. Hepatology 2013; 57: 1962-8.

9. Rautou PE, Douarin L, Denninger MH, Escolano S, Lebrec D, Moreau R, et al. Bleeding in patients with Budd-Chiari syndrome. J Hepatol 2011; 54: 56-63.

10. Wanless IR, Wong F, Blendis LM, Greig P, Heathcote EJ, Levy G. Hepatic and portal vein thrombosis in cirrhosis: possible role in development of parenchymal extinction and portal hypertension. Hepatology 1995; 21 : 1238-47.

11. Pan S, Kleppe LS, Witt TA, Mueske CS, Simari RD. The effect of vascular smooth muscle cell-targeted expression of tissue factor pathway inhibitor in a murine model of arterial thrombosis. Thromb Haemost 2004; 92: 495-502.

12. Mancuso A. Cirrhosis development probably arises from chronic microvascular ischemia. Med Hypotheses 2014; 82: 243-4. 
13 Mancuso A. The Ischemic Liver Cirrhosis Theory and its Clinical Implications. Med Hypotheses 2016; 94: 4-6.

14. Simonetto DA1, Yang HY, Yin M, de Assuncao TM, Kwon JH, Hilscher $\mathrm{M}$, et al. Chronic Passive Venous Congestion Drives Hepatic Fibrogenesis Via Sinusoidal Thrombosis and Mechanical Forces. Hepatology 2015; 61: 648-59.

15. Mancuso A. The target of TIPS for Budd-Chiari syndrome should be avoiding liver transplantation. Aliment Pharmacol Ther 2014; 39: 1342.

16. Mancuso A. TIPS for Budd-Chiari syndrome: Time to anticipate treatment. Liver Int 2014; 7: e325.

17. Mancuso A. Budd-Chiari Syndrome management: Timing of treatment is an open issue. Hepatology 2014; 59: 1213.

18. Plessier A, Sibert A, Consigny Y, Hakime A, Zappa M, Denninger MH, et al. Aiming at minimal invasiveness as a therapeutic strategy for BuddChiari syndrome. Hepatology 2006; 44: 1308-16.

19. Bilbao JI, Pueyo JC, Longo JM, Arias M, Herrero JI, Benito A, et al. Interventional therapeutic techniques in Budd-Chiari syndrome. Cardiovasc Intervent Radiol 1997; 20: 112-9.

20. Fisher NC, McCafferty I, Dolapci M, Wali M, Buckels JA, Olliff SP, et al. Managing Budd-Chiari syndrome: a retrospective review of percutaneous hepatic vein angioplasty and surgical shunting. Gut 1999; 44: 568-74.

21. Eapen CE, Velissaris D, Heydtmann M, Gunson B, Olliff S, Elias E. Favourable medium term outcome following hepatic vein recanalisation and/or transjugular intrahepatic portosystemic shunt for Budd Chiari syndrome. Gut 2006; 55: 878-84.

22. Perelló A, García-Pagán JC, Gilabert R, Suárez Y, Moitinho E, Cervantes $\mathrm{F}$, et al. TIPS is a useful long-term derivative therapy for patients with Budd-Chiari syndrome uncontrolled by medical therapy. Hepatology 2002; 35: 132-9.

23. Mancuso A, Fung K, Mela M, Tibballs J, Watkinson A, Burroughs AK, et al. TIPS for acute and chronic Budd-Chiari syndrome: a single-centre experience. J Hepatol 2003; 38: 751-4.

24. Rössle M, Olschewski M, Siegerstetter V, Berger E, Kurz K, Grandt D. The Budd-Chiari syndrome: outcome after treatment with the transjugular intrahepatic portosystemic shunt. Surgery 2004; 135: 394-403.

25. Mancuso A, Watkinson A, Tibballs J, Patch D, Burroughs AK. BuddChiari syndrome with portal, splenic, and superior mesenteric vein thrombosis treated with TIPS: who dares wins. Gut 2003; 52: 438.

26. Darwish Murad S, Valla DC, De Groen PC, Zeitoun G, Haagsma EB, Kuipers EJ, et al. Pathogenesis and treatment of Budd-Chiari syndrome combined with portal vein thrombosis. Am J Gastroenterol 2006; 101: 83-90.

27. Garcia-Pagán JC, Heydtmann M, Raffa S, Plessier A, Murad S, Fabris F, et al. TIPS for Budd-Chiari syndrome: long-term results and prognostics factors in 124 patients. Gastroenterology 2008; 135: 808-15.

28. Qi X, Guo W, He C, Zhang W, Wu F, Yin Z, et al. Transjugular intrahepatic portosystemic shunt for Budd-Chiari syndrome: techniques, indications and results on 51 Chinese patients from a single centre. Liver Int 2014; 34: 1164-75.

29. Tripathi D, Macnicholas R, Kothari C, Sunderraj L, Al-Hilou H, Rangarajan $\mathrm{B}$, et al. Good clinical outcomes following transjugular intrahepatic portosystemic stent-shunts in Budd-Chiari syndrome. Aliment Pharmacol Ther 2014; 39: 864-72.

30. Ringe B, Lang H, Oldhafer KJ, Gebel M, Flemming P, Georgii A, et al. Which is the best surgery for Budd-Chiari syndrome: venous decompression or liver transplantation? A single-center experience with 50 patients. Hepatology 1995; 21: 1337-44.

31. Hemming AW, Langer B, Greig P, Taylor BR, Adams R, Heathcote EJ. Treatment of Budd-Chiari syndrome with portosystemic shunt or liver transplantation. Am J Surg 1996; 171: 176-80.
32. Orloff MJ, Isenberg JI, Wheeler HO, Daily PO, Girard B. Budd-Chiari syndrome revisited: 38 years' experience with surgical portal decompression. J Gastrointest Surg 2012; 16: 286-300.

33. Zhang Y, Zhao H, Yan D, Xue H, Lau WY. Superior mesenteric veincaval-right atrium $Y$ shunt for treatment of Budd-Chiari syndrome with obstruction to the inferior vena cava and the hepatic veins-a study of 62 patients. J Surg Res 2011; 169: e93-9.

34. Srinivas BC, Dattatreya PV, Srinivasa KH, Prabhavathi CN, Manjunath $\mathrm{CN}$. Inferior vena cava obstruction: long-term results of endovascular management. Indian Heart J 2012;64:162-9.

35. Mancuso A, Martinelli L, De Carlis L, Rampoldi AG, Magenta G, Cannata A, et al. A caval homograft for Budd-Chiari syndrome due to inferior vena cava obstruction. World J Hepatol 2013; 27: 292-5.

36. Halff G, Todo S, Tzakis AG, Gordon RD, Starzl TE. Liver transplantation for the Budd-Chiari syndrome. Ann Surg 1990; 211: 43-9.

37. Rao AR, Chui AK, Gurkhan A, Shi LW, Al-Harbi I, Waugh R, et al. Orthotopic liver transplantation for treatment of patients with Budd-Chiari syndrome: a Single-center experience. Transplant Proc 2000; 32: 2206-7.

38. Ulrich F, Steinmüller T, Lang M, Settmacher U, Müller AR, Jonas S, et al. Liver transplantation in patients with advanced Budd-Chiari syndrome. Transplant Proc 2002; 34: 2278.

39. Srinivasan P, Rela M, Prachalias A, Muiesan P, Portmann B, Mufti GJ, et al. Liver transplantation for Budd-Chiari syndrome. Transplantation 2002; 73: 973-7.

40. Mentha G, Giostra E, Majno PE, Bechstein WO, Neuhaus P, O'Grady J, et al. Liver transplantation for Budd-Chiari syndrome: A European study on 248 patients from 51 centres. J Hepatol 2006; 44: 520-8.

41. Segev DL, Nguyen GC, Locke JE, Simpkins CE, Montgomery RA, Maley WR, et al. Twenty years of liver transplantation for Budd-Chiari syndrome: a national registry analysis. Liver Transpl 2007; 13: 1285-94.

42. Potthoff A, Attia D, Pischke S, Mederacke I, Beutel G, Rifai K, et al. Longterm Outcome of Liver Transplant Patients with Budd-Chiari Syndrome Secondary to Myeloproliferative Neoplasms. Liver Int 2015; 35: 2042-9.

43. Mancuso A. Time to resize the role of liver transplant for Budd-Chiari syndrome. Liver Int 2015; 35: 2339.

44. Choi GS, Park JB, Jung GO, Chun JM, Kim JM, Moon JI, et al. Living donor liver transplantation in Budd-Chiari syndrome: a single-center experience. Transplant Proc 2010; 42: 839-42.

45. Darwish Murad S, Plessier A, Hernandez-Guerra M, Fabris F, Eapen CE, Bahr MJ, et al. Etiology, management, and outcome of the Budd-Chiari syndrome. Ann Intern Med 2009; 151: 167-75.

46. Qi X, Ren W, Wang Y, Guo X, Fan D. Survival and prognostic indicators of Budd-Chiari sybdrome: a systematic review of 79 studies. Expert Rev Gastroenterol Hepatol 2015; 9: 865-75.

47. Mancuso A. Management of Budd-Chiari syndrome in children: same debated issues as in adults. Eur J Gastroenterol and Hepatol 2015: 27: 107-8.

48. Mancuso A. Outcome of Budd-Chiari syndrome in Behçet's syndrome. Semin Arthritis Rheum 2015; 45: e1.

49. He F, Zhao H, Dai S, Wu Y, Wang L, Huang H, et al. Transjugular intrahepatic portosystemic shunt for Budd-Chiari syndrome with diffuse occlusion of hepatic veins. Sci Rep 2016; 6: 36380 .

50. Rosenqvist K, Sheikhi R, Eriksson LG, Rajani R, Rorsman F, Sangfelt P, et al. Endovascular treatment of symptomatic Budd-Chiari syndrome in favour of early transjugular intrahepatic portosystemic shunt. Eur J Gastroenterol Hepatol 2016; 28: 656-60.

How to cite this article: Mancuso A. Timing of transjugular intrahepatic portosystemic shunt for Budd-Chiari syndrome: An Italian hepatologist's perspective. J Transl Intern Med 2017; 5: 194-99. 